\title{
Programme de collaboration franco-suisse pour la création de nouvelles variétés de vigne durablement résistantes au mildiou et à l'oïdium
}

\section{French-Swiss collaborative program for breeding new grapevine varieties durably resistant to downy and powdery mildew}

\author{
C. Schneider ${ }^{1}$, J.-L. Spring ${ }^{2}$, C. Onimus ${ }^{1}$, E. Prado ${ }^{1}$, T. Verdenal ${ }^{2}$, G. Lemarquis ${ }^{1}$, F. Lorenzini ${ }^{2}$, L. Ley ${ }^{1}$, P. Duruz ${ }^{2}$, \\ K. Gindro ${ }^{2}$ et D. Merdinoglu ${ }^{1}$ \\ ${ }^{1}$ INRA, Université de Strasbourg, UMR 1131 SVQV, 68000-Colmar, France \\ ${ }^{2}$ Agroscope, 1260 Nyon, Suisse
}

\begin{abstract}
Résumé. Une alternative aux traitements phytosanitaires pour lutter contre le mildiou et l'oïdium de la vigne est le développement de variétés résistantes. Leur création doit intégrer non seulement le niveau de résistance mais également sa durabilité, ainsi que les performances agronomiques notamment la productivité et la composition des baies qui doit convenir à la production de vins de haute qualité. L'Inra (France) a engagé le programme de sélection ResDur, basé sur le pyramidage des facteurs de résistance, en 2000. Il a conduit à l'inscription au catalogue français d'une première série de variétés à résistance polygénique Artaban, Floreal, Vidoc, Voltis - en 2018. Agroscope (Suisse) a démarré la création de variétés résistantes en 1996, en mobilisant les résistances portées par des variétés allemandes, principalement Bronner. Un premier aboutissement a été l'homologation en Suisse de Divico et Divona, respectivement en 2013 et 2018.

En 2009 l'Inra et Agroscope ont démarré un programme de sélection commun, visant à combiner les facteurs de résistance aux maladies présents de manière complémentaire dans leurs lignées respectives. Les croisements réalisés ont généré, après sélection assistée par marqueurs, 400 descendants porteurs des facteurs de résistance Rpv1, Rpv10 et/ou Rpv3 ; Run1, Ren3 et/ou Ren3.2. Leur phénotypage agronomique et œnologique est réalisé dans le cadre d'un réseau d'essais situés dans les deux Instituts à Colmar (FR) et à Pully $(\mathrm{CH})$. Les premières observations pluriannuelles, portant sur 80 descendants, ont permis d'étudier leur stabilité phénotypique pour les caractères de résistance, les traits culturaux et la qualité des vins. A l'issue de cette étape, quinze variétés candidates ont été sélectionnées et installées en essais d'évaluation de leur valeur agronomique, technologique et environnementale (VATE) en Valais (CH), ainsi que dans trois régions françaises (Champagne, Val de Loire, Vallée du Rhône). L'inscription au catalogue des premières co-obtentions Inra/Agroscope est prévue à l'horizon 2024-2025.
\end{abstract}

\begin{abstract}
An alternative promising solution to chemical treatments for grapevine protection against downy and powdery mildew is the development of resistant cultivars. The creation of such cultivars needs to take into account both of resistance efficacy and durability and agronomic performances, in particular for productivity and berry traits, which must be suitable for the production of high quality wines. Inra (France) has launched the ResDur breeding program, based on pyramiding of resistance factors, in 2000. Originating from this program, a first series of varieties with polygenic resistance to downy and powdery mildew - Artaban, Floreal, Vidoc and Voltis - were already registered in 2018. Agroscope has started breeding for resistance in 1996, by mobilizing the resistance from German varieties, mainly Bronner. A first achievement was the approval in Switzerland of Divico and Divona, respectively in 2013 and 2018.

Agroscope and Inra have launched a partnership in 2009, in order to develop a shared breeding program and to combine the disease resistance factors carried in a complementary way by their respective breeding lines. The undertaken cross-breedings resulted, after marker assisted selection, in 400 offspring carrying the resistance factors Rpv1, Rpv10 and/or Rpv3; Run1, Ren3 and/or Ren3.2. Their agronomic and oenological phenotyping is achieved through a network of field trials located in both institutes, in Colmar (FR) and Pully (CH). The first multi-annual field observations realized on 80 individuals permitted to study their phenotypic stability for resistance, agronomic, and wine quality traits. Finally, 15 candidate varieties were selected and are currently under Value for Cultivation and Use (VCU) experimentation in Valais $(\mathrm{CH})$ and in three French regions (Champagne, Loire Valley, Rhone Valley). The registration of the first varieties developed by INRA and Agroscope as co-owners is expected in 2024-2025.
\end{abstract}

(C) The Authors, published by EDP Sciences. This is an Open Access article distributed under the terms of the Creative Commons Attribution License 4.0 (http://creativecommons.org/licenses/by/4.0/). 
La lutte contre les maladies fongiques de la vigne, en particulier le mildiou et l'oïdium qui avec la pourriture grise sont les plus dommageables, reste essentiellement assurée par l'utilisation de fongicides. Cette méthode est efficace pour maîtriser les pertes de récolte, tant en quantité qu'en qualité. Elle est cependant relativement onéreuse et elle se traduit par des effets négatifs sur la santé humaine [1] et l'environnement qui peuvent affecter l'image du vin.

Une illustration de la quantité de produits phytosanitaires utilisés par le secteur viticole en France est donnée dans le Tableau 1. Dans l'absolu, en considérant les tonnages de substances actives (SA) consommés, il apparait une tendance à la diminution sur la période 19992009. Mais cette baisse reflète la réduction des surfaces en vigne et des doses/hectare homologuées. En proportion de la consommation totale, le secteur de la viticulture reste l'un des plus gros consommateurs, avec environ un quart des produits phytopharmaceutiques consommés, alors qu'il ne représente que $3 \%$ de la SAU $[2,3]$ (Tableau 1).

\section{De nouvelles variétés résistantes pour réduire l'utilisation des produits phytosanitaires}

Aujourd'hui, réduire l'usage des pesticides est un enjeu majeur pour développer une agriculture et notamment une viticulture durable et de qualité. C'est pourquoi les politiques au niveau européen ou national, tant en France qu'en Suisse, cherchent à mettre en place des dispositifs incitatifs pour la réduction des intrants phytosanitaires en agriculture. Il s'agit de maintenir la performance économique des filières, en y ajoutant un niveau élevé de performances environnementales et sociales. En France, ces objectifs sont déclinés dans le plan Ecophyto, au travers de la mise en place de certificats d'économie de produits phytopharmaceutiques (CEPP) [4]. En Suisse, des développements techniques de transmission de l'information, ainsi que des contributions incitatives sont également mises en œuvre, visant la réduction de l'utilisation d'intrants phytosanitaires. Parmi les voies possibles pour atteindre ces objectifs, l'utilisation des résistances génétiques naturelles aux agents pathogènes constitue une voie prometteuse. Elle repose sur l'amélioration génétique, souvent longue et fastidieuse, mais une fois les variétés adaptées développées, leur utilisation est facile et peu onéreuse.

Chez la vigne, plusieurs sources de résistance aux maladies ont été identifiées au sein d'espèces de Vitis d'origine américaine ou asiatique [5-7]. Ces vignes sauvages sont génétiquement proches de la vigne cultivée et peuvent transmettre leurs caractères de résistance par voie de croisements. L'analyse génétique de ces caractères a énormément progressé au cours des 20 dernières années, et de nombreux facteurs de résistance au mildiou ou à l'oïdium ont pu être identifiés et positionnés sur le génome. Une liste compilée avec les références est disponible sur www.vivc.de (data on breeding and genetics). Il faut cependant savoir que ces facteurs de résistance ne sont pas tous pertinents ou faciles à utiliser dans un programme de création variétale, soit en raison d'un faible effet de protection, soit en raison d'un niveau d'introgression insuffisant dans le fonds génétique de la
Tableau 1. Consommation de produits phytopharmaceutiques (PPP) par la viticulture en France.

\begin{tabular}{|l|c|c|c|}
\hline & 1999 & 2003 & 2009 \\
\hline Surface, $\times 1000$ ha & 901 & 851 & 790 \\
\hline PPP, tonnes de SA $\left(^{*}\right)$ & 14100 & 10700 & 9800 \\
\hline PPP, \% toutes cultures $\left(^{*}\right)$ & 26 & 24 & 28 \\
\hline
\end{tabular}

(*) : Soufre minéral exclu ; $80 \%$ des PPP sont des fongicides.

Tableau 2. Facteurs de résistance au mildiou et à l'oïdium utilisés par les obtenteurs européens.

\begin{tabular}{|c|c|c|c|c|}
\hline $\begin{array}{c}\text { Maladie (agent } \\
\text { pathogène) }\end{array}$ & $\begin{array}{c}\text { Facteur de } \\
\text { résistance }\end{array}$ & Origine & $\begin{array}{c}\text { Situation } \\
\text { sur le } \\
\text { génome }\end{array}$ & $\begin{array}{c}\text { Niveau de } \\
\text { protection } \\
\text { conféré }\end{array}$ \\
\hline $\begin{array}{c}\text { Mildiou } \\
\text { (Plasmopara } \\
\text { viticola) }\end{array}$ & $R p v 1$ & V. rotundifolia & chr.12 & élevé \\
\cline { 2 - 5 } & $R p v 3$ & V. rupestris & chr. 18 & moyen \\
\cline { 2 - 5 } & $R p v 10$ & V. amurensis & chr.9 & élevé \\
\cline { 2 - 5 } & $R p v 12$ & V. amurensis & chr.14 & élevé \\
\hline \multirow{2}{*}{$\begin{array}{c}\text { Ö̈dium } \\
\text { (Erysiphe } \\
\text { necator) }\end{array}$} & Run1 & V. rotundifolia & chr.12 & total \\
\cline { 2 - 5 } & $R e n 1$ & V. vinifera & chr.13 & élevé \\
\cline { 2 - 5 } & Ren3 & V. rupestris & chr.15 & moyen \\
\hline
\end{tabular}

vigne cultivée (Vitis vinifera). Finalement, seuls trois ou quatre facteurs de résistance sont réellement mobilisés dans les programmes de création variétale poursuivis par les obtenteurs européens (Tableau 2).

Des facteurs de résistance identifiés plus récemment sont en cours d'introgression et viendront progressivement compléter le panel de facteurs de résistance utilisable par les sélectionneurs.

\section{Une stratégie Inra-Agroscope partagée pour accroître la durabilité des résistances}

Les facteurs de résistance au mildiou et à l'oïdium identifiés par les travaux de cartographie sont en majorité situés dans des régions génomiques riches en gènes de résistance canoniques, appelés gènes $R$ [8]. Cela indique que les facteurs de résistance sont probablement impliqués dans une interaction gène pour gène avec l'agent pathogène, et que leur efficacité est liée à cette correspondance très spécifique. Si une mutation affecte le motif moléculaire du côté de l'agent pathogène, la correspondance est rompue et le gène de résistance perd son efficacité. Il s'agit alors d'un contournement de résistance par une souche virulente du pathogène.

\subsection{Des cas de contournements de la résistance déjà observés chez la vigne}

De nombreux cas de contournements de gènes- $R$ ont déjà été observés pour divers patho-systèmes. Chez la vigne, un premier cas a été décrit pour la résistance au mildiou de Bianca, déterminée par le facteur Rpv3 [9]. Des travaux plus récents ont mis en évidence un cas 
similaire pour le facteur de résistance à l'oïdium Runl, avec un isolat virulent originaire du Sud-Est des EtatsUnis [10]. Ces résultats montrent que la question de la durabilité est aussi importante que celle du niveau des résistances, en particulier dans le cas d'une espèce pérenne. Il apparaît en fin de compte que les facteurs de résistance utilisables en sélection constituent une ressource limitée et que leur effet de protection peut être remis en cause par l'apparition de souches contournantes. Etant donné que l'incorporation d'un facteur de résistance dans le fonds génétique cultivé est par ailleurs longue et onéreuse, il parait indispensable de prendre en compte la durabilité des résistances dans la stratégie de sélection des nouveaux cultivars [11]. La combinaison de plusieurs facteurs de résistance à une maladie au sein d'une même variété permet de répondre à cette exigence, conformément à une résolution de l'OIV adoptée en 2013 [12]. Le développement de nouveaux outils, telle la sélection assistée par marqueurs (SAM), autorise la mise en œuvre de ce type de stratégie, qui permet d'obtenir des variétés à résistances polygéniques [13].

\subsection{Une complémentarité entre les obtentions résistantes antérieures de l'Inra et de Agroscope}

L'Inra a engagé son programme ResDur en 2000, avec l'ambition de développer une gamme de variétés durablement résistantes au mildiou et à l'oïdium adaptée aux grands bassins viticoles français [14]. Ce programme repose sur l'utilisation de géniteurs de résistance complémentaires, dont des lignées d'introgression issues de $V$. rotundifolia par rétrocroisements successifs [15] constituent le pivot. Il met en œuvre un schéma de sélection organisé en trois étapes : (i) sélection précoce basée sur le tri des descendances par SAM afin de ne retenir que les individus pyramidés pour les facteurs de résistance portés par les deux parents ; (ii) sélection intermédiaire appuyée sur un réseau d'essais au sein des Unités Expérimentales de l'INRA (Colmar, Bordeaux, Angers, Montpellier) ; (iii) sélection finale dans le réseau d'essais de Valeur Agronomique, Technologique et Environnementale (VATE), de portée nationale, en collaboration avec l'Institut Français de la Vigne et du Vin (IFV). Les deux dernières étapes portent sur l'évaluation de l'ensemble des aptitudes culturales et sur la qualité sensorielle des vins, à partir de vinifications en très petits ou petits volumes. La durée totale du processus de sélection est d'environ 15 ans.

Un premier aboutissement de ce programme a été l'inscription au catalogue français des variétés - Artaban, Floreal, Vidoc, Voltis - en 2018. Leurs principales caractéristiques sont présentées dans des fiches techniques accessibles sur http://presse.inra.fr/Communiquesde-presse/Sival-d-or-2019. Ces variétés découlent de croisements entre des obtentions "Bouquet", rétrocroisements d'ordre 4 (RV4) ou 5 (RV5) de $V$. rotundifolia, et des variétés du Julius Kühn Institut (Siebeldingen, Allemagne) principalement Regent. Leur résistance au mildiou est élevée, déterminée par deux facteurs de résistance $(R p v 1 \& R p v 3)$ et leur résistance à l'oïdium totale, également déterminée par deux facteurs de résistance (Run1 \& Ren3). Elles répondent en cela aux recommandations de l'OIV [11]. Leur époque de maturité ( $2^{\text {ème }}$ époque) est compatible avec un déploiement dans la plupart des vignobles français, ainsi que dans d'autres régions viticoles. Leurs aptitudes culturales et œnologiques rejoignent les ambitions du plan Ecophyto pour la filière viticole, à savoir la réduction du recours aux produits phytosanitaires, tout en assurant un niveau de production élevé tant en quantité qu'en qualité.

Agroscope a démarré un programme de création variétale axé sur la résistance aux maladies cryptogamiques en 1996, afin de prolonger son programme principalement orienté vers l'obtention de variétés rouges de qualité peu sensibles à la pourriture grise (Botrytis cinerea). Dans une première phase, le Gamaret, choisi comme géniteur européen ( $V$. vinifera) pour son potentiel qualitatif et son exceptionnelle résistance à la pourriture, a été croisé avec des variétés du Weinbau Institut (Freiburg, Allemagne), porteuses de facteurs de résistance au mildiou et à l'oïdium provenant de vignes sauvages américaines et asiatiques, principalement Bronner. Des tests biochimiques, basés sur la synthèse des phytoalexines stilbéniques, ont été développés pour réaliser un tri précoce des descendances, relativement à la résistance au mildiou [16]. Les performances agronomiques et œnologiques sont évaluées dans les conditions représentatives du bassin lémanique, du Valais et du Tessin. Au total, la procédure de sélection dure un peu plus de 15 ans.

Ce programme a débouché sur l'homologation en Suisse des premières variétés résistantes aux principales maladies de la vigne, Divico [17] et Divona [18], respectivement en 2013 et 2018. Leur résistance au mildiou et à l'oïdium est élevée, avec en outre une très bonne résistance à la pourriture grise et une faible sensibilité au black rot. Nous avons vérifié que les facteurs de résistance portés par leur géniteur de résistance commun (Bronner) [19] ont bien été transmis aux deux variétés : Rpv10 pour le mildiou et Ren3.2 pour l'oïdium. Divico est une variété rouge relativement précoce, comparable au Gamaret, adaptée à la plupart des vignobles de Suisse. La précocité de Divona (blanc) est plus marquée et il faut lui réserver les situations les plus fraîches du vignoble. Les deux variétés permettent d'élaborer des vins dotés d'une complexité aromatique intéressante qui sont généralement très bien appréciés en dégustation.

Il apparaît en définitive que les obtentions respectives des deux instituts sont très complémentaires, que ce soit pour les facteurs de résistance au mildiou et à l'oïdium, pour d'autres traits de résistance, notamment vis-à-vis de la pourriture grise, ou enfin pour des traits culturaux comme la précocité ou la productivité.

\subsection{Le partenariat Inra-Agroscope : principes et réalisations}

Forts de leur expérience respective et de leurs premiers résultats, Inra et Agroscope ont décidé d'engager un partenariat en 2009, afin de conduire un programme commun de création de variétés de vigne résistantes aux maladies. Il a été convenu de mettre en commun les ressources constituées par les obtentions les plus avancées en sélection de part et d'autre, de réaliser des croisements et d'évaluer et trier les descendances dans un dispositif partagé et enfin de présenter les variétés sélectionnées à 
Tableau 3. Géniteurs utilisés dans le programme Inra-Agroscope.

\begin{tabular}{|c|c|c|c|c|}
\hline Institut & Géniteur & Parents & $\begin{array}{c}\text { Facteurs } \\
\text { de résist. } \\
\text { mildiou }\end{array}$ & $\begin{array}{c}\text { Facteurs } \\
\text { de résist. } \\
\text { oïdium }\end{array}$ \\
\hline \multirow{5}{*}{ Inra } & IJ 119 & $\begin{array}{l}\text { Mtp 3082-1-42 } \\
\text { x Regent }\end{array}$ & $\begin{array}{c}\text { Rpv1 \& } \\
\text { Rpv3 }\end{array}$ & $\begin{array}{l}\text { Run1 \& } \\
\text { Ren3 }\end{array}$ \\
\hline & $\begin{array}{l}\text { IJ } 134 \\
\text { (Artaban) }\end{array}$ & $\begin{array}{l}\text { Mtp 3082-1-42 } \\
\text { x Regent }\end{array}$ & $\begin{array}{c}\text { Rpv1 \& } \\
\text { Rpv3 }\end{array}$ & $\begin{array}{l}\text { Run1 \& } \\
\text { Ren3 }\end{array}$ \\
\hline & $\begin{array}{l}\text { Col-2007G } \\
\text { (Floreal) }\end{array}$ & $\begin{array}{c}\text { Mtp 3159-2-12 } \\
\text { x Villaris }\end{array}$ & $\begin{array}{c}\text { Rpv1 \& } \\
\text { Rpv3 }\end{array}$ & $\begin{array}{l}\text { Run1 \& } \\
\text { Ren3 }\end{array}$ \\
\hline & $\begin{array}{l}\text { Col-2011G } \\
\text { (Voltis) }\end{array}$ & $\begin{array}{l}\text { Mtp } 3159-2-12 \\
\times \text { Villaris }\end{array}$ & $\begin{array}{c}\text { Rpv1 \& } \\
\text { Rpv3 }\end{array}$ & $\begin{array}{l}\text { Run1 \& } \\
\text { Ren3 }\end{array}$ \\
\hline & Col-2024G & $\begin{array}{c}\text { Mtp } 3159-2-12 \\
\times \text { Villaris } \\
\end{array}$ & $\begin{array}{c}\text { Rpv1 \& } \\
\text { Rpv3 }\end{array}$ & $\begin{array}{l}\text { Run1 \& } \\
\text { Ren3 } \\
\end{array}$ \\
\hline \multirow{3}{*}{ Agroscope } & IRAC-1933 & $\begin{array}{l}\text { Bronner } x \\
\text { Cornalin }\end{array}$ & Rpv10 & Ren3.2 \\
\hline & $\begin{array}{l}\text { IRAC-2060 } \\
\text { (Divona) }\end{array}$ & $\begin{array}{c}\text { Gamaret } x \\
\text { Bronner }\end{array}$ & Rpv10 & Ren3.2 \\
\hline & $\begin{array}{l}\text { IRAC-2091 } \\
\text { (Divico) }\end{array}$ & $\begin{array}{l}\text { Gamaret x } \\
\text { Bronner }\end{array}$ & Rpv10 & Ren3.2 \\
\hline
\end{tabular}

l'inscription et d'attribuer les droits d'obtenteurs attachés aux certificats d'obtention végétale (COV) aux deux instituts.

Pour la sélection des descendances, nous nous sommes accordés sur les principes retenus pour le programme InraResDur : (i) Sélection précoce par SAM des seuls individus porteurs d'au moins deux facteurs de résistance au mildiou, dont $R p v 1$, et deux facteurs de résistance à l'oïdium, dont Run1. (ii) Sélection intermédiaire basée sur les deux sites d'expérimentation de Colmar (FR) pour l'Inra et de Pully $(\mathrm{CH})$ pour Agroscope. (iii) Sélection finale appuyée sur un réseau multi-sites franco-suisse, ce qui nécessitait une adaptation du règlement technique d'inscription français.

Les croisements réalisés dans le cadre du programme ont systématiquement mobilisé un géniteur Inra et un géniteur Agroscope, parmi ceux listés dans le Tableau 3. Ils sont identifiés par leur référence d'obtenteur et par leur dénomination en cas d'inscription /homologation.

Trois campagnes de croisements ont permis de générer plusieurs milliers de descendants, avec des effectifs très variables selon les populations. Parmi les facteurs de réussite, on retiendra l'aptitude à la castration et à la nouaison du parent femelle, la qualité du pollen, ainsi que les conditions de pollinisation et de nouaison. Les quatre populations les plus nombreuses sont présentées dans le Tableau 4. Ce tableau donne également le nombre d'individus pyramidés, résultant du tri par SAM en sélection précoce, pour chacune des populations. Relativement au nombre de pépins, leur proportion va de 8 à $13 \%$, pour un taux attendu de $9 / 64^{\text {ème }}$ dans l'hypothèse d'un taux de germination des pépins de $50 \%$. Les écarts sont principalement dus aux taux de germination réels qui fluctuent selon les populations.

Il faut trois années pour réaliser le croisement, la sélection précoce par SAM et l'élevage en serre des pieds de semis retenus et enfin la multiplication par greffe-bouture pour produire les plants nécessaires aux expérimentations de terrain. L'installation des premiers dispositifs de sélection intermédiaire a ainsi été réalisée en 2012, conjointement sur les sites de Colmar et de Pully.
Tableau 4. Descendances les plus importantes du programme Inra-Agroscope.

\begin{tabular}{|c|c|c|c|c|}
\hline Population & $\begin{array}{c}\text { Année du } \\
\text { croisement }\end{array}$ & $\begin{array}{c}\text { Nombre de } \\
\text { pépins }\end{array}$ & $\begin{array}{c}\text { Nombre } \\
\text { d'individus } \\
\text { pyramidés }\end{array}$ & $\begin{array}{c}\text { Taux en \% } \\
\text { du nombre } \\
\text { de pépins }\end{array}$ \\
\hline $\begin{array}{c}\text { IRAC-1933 x } \\
\text { Col-2011G }\end{array}$ & 2009 & 350 & 29 & 8 \\
\hline $\begin{array}{c}\text { IRAC-1933 x } \\
\text { Col-2024G }\end{array}$ & 2009 & 460 & 42 & 9 \\
\hline $\begin{array}{c}\text { IJ 119 } x \\
\text { IRAC 2060 }\end{array}$ & 2012 & 520 & 70 & 13 \\
\hline $\begin{array}{c}\text { IJ 134 } x \\
\text { IRAC 2091 }\end{array}$ & 2014 & 1900 & 190 & 10 \\
\hline
\end{tabular}

\section{Les premiers résultats de l'étape de sélection intermédiaire}

Les dispositifs de sélection intermédiaire englobent les modalités variétales suivantes : deux variétés témoins bien connues, Chardonnay et Merlot, et l'ensemble des individus pyramidés, pour une évaluation comparative. Dans un essai donné, le porte-greffe, la conduite des souches et les opérations culturales sont identiques, à l'exception des traitements phytosanitaires. Les variétés témoins bénéficient d'une protection fongicide conventionnelle, alors que les individus à évaluer n'en reçoivent aucune. Pour faciliter l'exécution des traitements, le dispositif est scindé en deux parties séparées par un rang de garde. Afin de mesurer la pression de maladies, les variétés témoins sont également implantées dans la zone sans protection fongicide, ces parcelles élémentaires ne servant qu'aux notations de maladies.

L'essai de Pully a été mis en place en 2012, avec des micro-parcelles de 5 pieds par génotype. Les souches, greffées sur 3309C sont conduites en Guyot simple $(200 \times 85 \mathrm{~cm})$. Situé dans le Bassin lémanique à $460 \mathrm{~m}$ d'altitude, ce site est caractérisé par des précipitations annuelles moyennes de $1140 \mathrm{~mm}$ et une température moyenne d'avril à septembre de $15^{\circ} \mathrm{C}$. Le sol est formé de colluvions fertiles. La pression mildiou y est assez forte et extrêmement élevée pour l'oïdium.

L'essai de Colmar, également mis en place en 2012, met en œuvre des micro-parcelles de 4 pieds par génotype. Les souches, greffées sur SO4, sont conduites en guyot double $(170 \times 140 \mathrm{~cm})$. Situé dans la plaine d'Alsace à $210 \mathrm{~m}$ d'altitude, les précipitations annuelles atteignent en moyenne $620 \mathrm{~mm}$ et la température moyenne d'avril à septembre $16.5^{\circ} \mathrm{C}$. Le sol est formé de lœss très fertiles et les pressions mildiou et oïdium y sont généralement élevées.

Les résultats présentés correspondent, sauf indication contraire, à la moyenne de trois années d'observations, allant de 2014 à 2016, pour les 79 individus pyramidés sélectionnés parmi les populations de 2009.

\subsection{Le niveau de résistance aux maladies des individus pyramidés}

Pour la résistance au mildiou, les facteurs de résistance associent Rpv1 à Rpv3 et/ou Rpv10. Chacun détermine une résistance partielle, de niveau moyen à élevé. Comme attendu, leur pyramidage ne conduit pas à une résistance totale, mais permet d'atteindre un très haut niveau de résistance, comme il apparait sur la Fig. 1. Pour une 


\section{Mildiou}

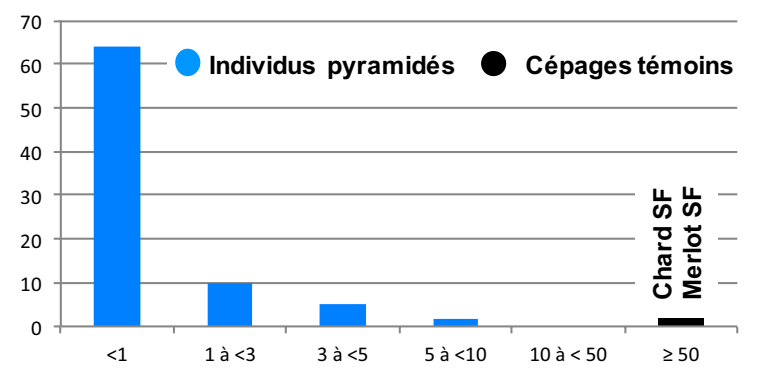

Figure 1. Distribution des individus selon l'intensité des dégâts de mildiou sur feuilles, en $\%$ de la surface foliaire, au stade véraison (Moyenne 2014-2016, valeur maximale observée à Colmar ou Pully, absence d'application de fongicides, y compris pour les témoins Chardonnay et Merlot).

large majorité de descendants, les dégâts restent limités à environ $1 \%$ de la surface foliaire, pour les 3 années et les 2 sites. En comparaison, les deux cépages témoins sont fortement impactés, avec des dégâts allant jusqu'à $90 \%$ en 2016.

La résistance à l'oïdium fait intervenir les facteurs Runl et Ren3, qui peut être apporté par les géniteurs de l'Inra et/ou de l'Agroscope. Dans le dernier cas, il provient de Bronner et est noté Ren3.2 (cf. Tableau 3). Tous les individus pyramidés étant porteurs de Runl, conférant une résistance totale, aucun symptôme d'oïdium n'a pu être observé, ni sur feuilles ni sur grappes, quel que soit le lieu ou l'année. Pour cette maladie, Runl masque l'effet des autres facteurs, le pyramidage se justifiant alors par l'objectif de durabilité de la résistance.

Relativement peu de travaux ont été consacrés au déterminisme génétique de la résistance au black rot et les connaissances sont encore partielles [20]. Agroscope a néanmoins observé dans ses descendances issues de Bronner une proportion non négligeable d'individus peu à très peu sensibles. Ce caractère a été pris en compte dans les critères de sélection et est présent dans les géniteurs d'Agroscope utilisés dans le programme commun avec l'Inra. Nous avons pu vérifier dans le dispositif de sélection intermédiaire qu'il se transmettait bien aux nouvelles descendances selon un mode qui pourrait correspondre à l'existence d'un facteur de résistance partielle a effet dominant. La Fig. 2 illustre cet aspect, avec une distribution bimodale des individus pour les dégâts de black rot observés à Colmar, où la pression est régulièrement assez forte. Les observations ont aussi été réalisées dans le dispositif de Pully, mais la pression du black rot y est faible et les résultats peu informatifs.

\subsection{Aspects agronomiques}

\subsubsection{Phénologie}

Les génotypes étudiés ont présenté une très grande variabilité en ce qui concerne la précocité de maturation. Les dates de vendange se sont échelonnées selon les individus entre le 15 septembre et le 23 octobre à Colmar et entre le 9 septembre et le 16 octobre à Pully, en moyenne des trois années de mesure. La date moyenne, tous individus confondus, est le 6 octobre à Colmar et le 29 septembre à Pully. La cohérence entre les deux sites est très bonne pour ce paramètre. Pour les 15 variétés
Black rot

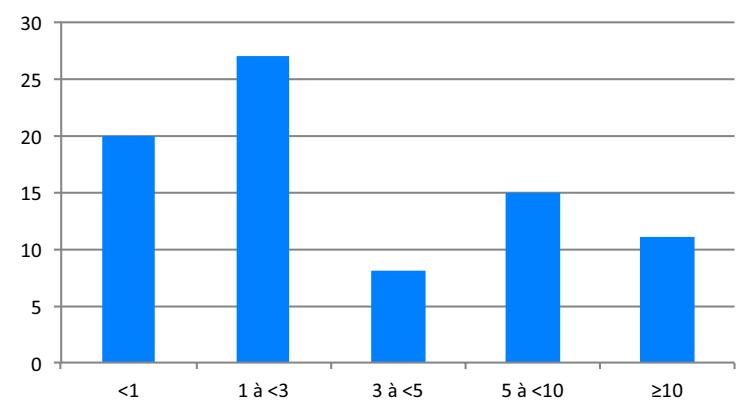

Figure 2. Distribution des individus selon l'intensité des dégâts de blackrot sur baies, en \% de baies nécrosées, au stade véraison (Moyenne 2014-2016, valeur observée à Colmar, absence d'application de fongicides).

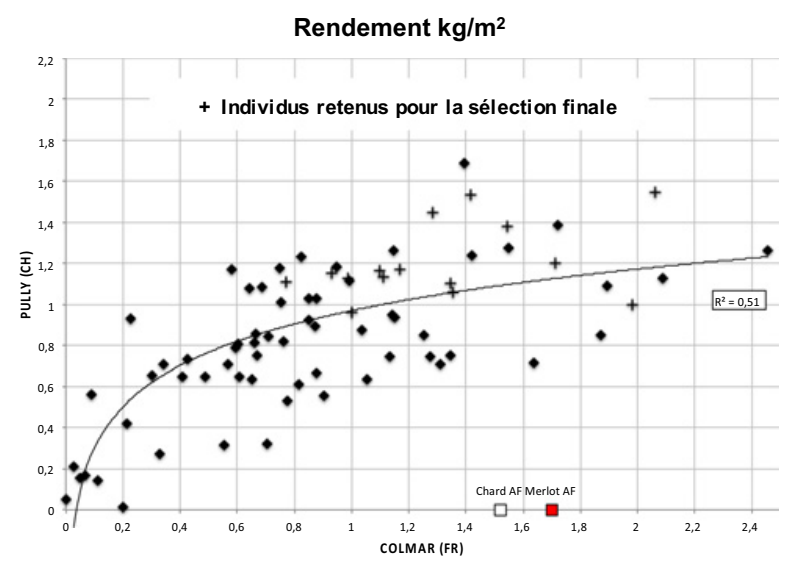

Figure 3. Relation entre les rendements enregistrés à Colmar et à Pully (Moyenne 2014-2016, individus 2009 sans protection fongicides $(\mathrm{SF})$, cépages témoins avec protection fongicides(AF)).

candidates retenues pour l'étape de sélection finale, la fourchette s'étend du 17 septembre au 18 octobre à Colmar et du 10 septembre au 14 octobre à Pully. Trois d'entre elles sont de maturité précoce, sept mûrissent entre la première et la deuxième époque et cinq sont de maturité tardive (deuxième époque tardive à troisième époque) selon Pulliat.

\subsubsection{Potentiel de production}

La Fig. 3 présente la production moyenne des 79 génotypes sur les sites de Pully et de Colmar. Il apparaît que la réponse en terme de rendement total exprimé en $\mathrm{kg} / \mathrm{m}^{2}$ présente une bonne cohérence entre les deux sites expérimentaux. Les valeurs moyennes sont de $0.9 \mathrm{~kg} / \mathrm{m}^{2}$ à Pully et $1.0 \mathrm{~kg} / \mathrm{m}^{2}$ à Colmar. A ce titre il faut relever que l'essai a été conduit sans régulation de la charge à Colmar alors qu'une légère réduction de la récolte a été entreprise avant véraison à Pully sur les génotypes présentant apparemment un potentiel de production supérieur à $1.5 \mathrm{~kg} / \mathrm{m}^{2}$. Cette régulation de la récolte n'a touché qu'un peu plus d'un tiers des candidats. La variation des rendements entre génotypes est considérable puisqu'elle va de la quasi stérilité à des niveaux de rendements supérieurs à $2.4 \mathrm{~kg} / \mathrm{m}^{2}$. Si l'on considère les 15 candidats les plus intéressants retenus pour la sélection finale, la 


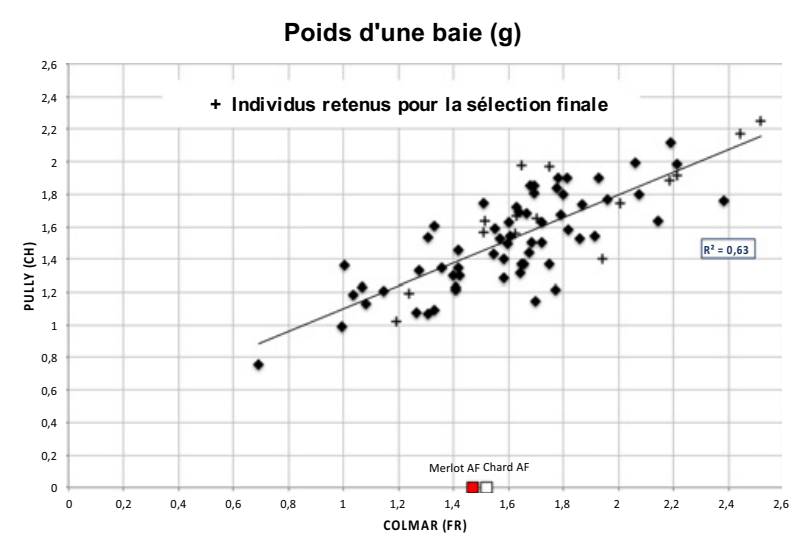

Figure 4. Relation entre les poids unitaires de la baie enregistrés à Colmar et à Pully (Moyenne 2014-2016, individus 2009 SF, cépages témoins $\mathrm{AF}$ ).

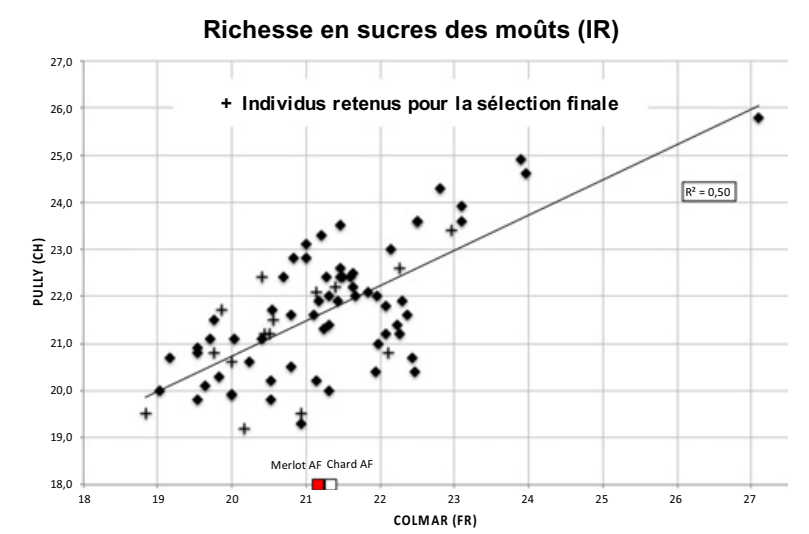

Figure 5. Relation entre l'indice réfractométrique du moût à la récolte, à Colmar et à Pully (Moyenne 2014-2016, individus 2009 SF, cépages témoins AF).

moyenne s'établit à $1.2 \mathrm{~kg} / \mathrm{m}^{2}$ à Pully et $1.3 \mathrm{~kg} / \mathrm{m}^{2}$ à Colmar, avec une fourchette variant de 1 à $2 \mathrm{~kg} / \mathrm{m}^{2}$.

La Fig. 4 illustre la relation entre les poids d'une baie constatés à Colmar et Pully. La relation peut être qualifiée de très étroite et confirme la bonne cohérence des observations effectuées sur les deux sites expérimentaux. Les baies sont légèrement plus lourdes à Colmar $(1.7 \mathrm{~g})$ qu'à Pully (1.6g). Pour les 15 génotypes retenus ces valeurs s'établissent respectivement à $1.3 \mathrm{~g}$ (Colmar) et $1.2 \mathrm{~g}$ (Pully).

\subsubsection{Composition des moûts}

Les valeurs de l'indice réfractométrique des moûts déterminé à la vendange à Colmar et à Pully sont reportées dans la Fig. 5. Ici également on peut relever la très bonne cohérence des résultats entre les deux sites. Les valeurs moyennes s'élèvent à 21.2\% Brix à Colmar et à $21.7 \%$ Brix à Pully avec des extrêmes situés entre $18.8 \%$ Brix et $27.1 \%$ Brix. Pour les 15 génotypes retenus ces valeurs sont de 20.8\% Brix à Colmar et de $21.2 \%$ Brix à Pully.

Les valeurs de $\mathrm{pH}$ des moûts (Fig. 6) présentent une relation un peu moins étroite entre les deux sites. Le $\mathrm{pH}$ moyen contrôlé à Colmar (3.17) est un peu plus élevé que celui constaté à Pully (3.10). Les variations entre génotypes sont relativement importantes.

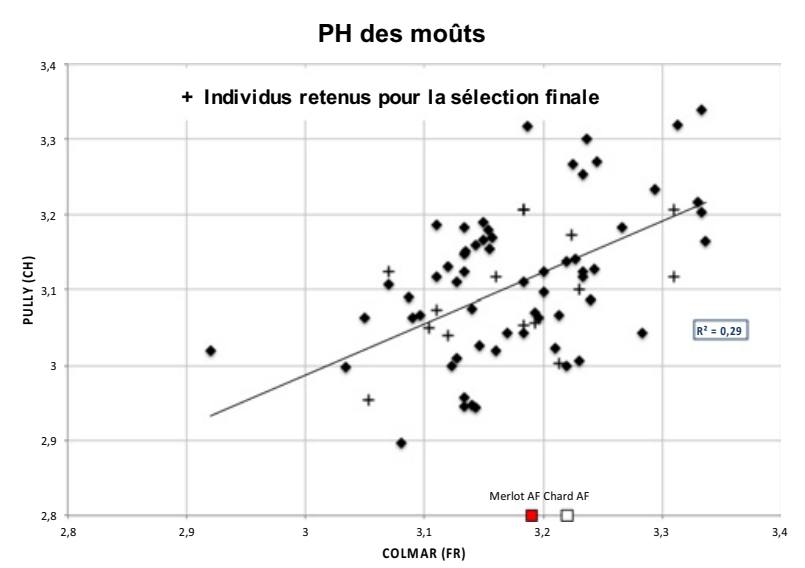

Figure 6. Relation entre le $\mathrm{pH}$ du moût à la récolte, à Colmar et à Pully (Moyenne 2014-2016, individus 2009 SF, cépages témoins AF).

\subsection{La qualité des vins}

Les raisins produits au niveau du dispositif de sélection intermédiaire sont vinifiés à très petite échelle (2 à 5 litres) à Colmar et à Pully, selon un procédé standardisé propre à chaque site. Cette opération est réservée aux individus dont la production atteint au moins $3 \mathrm{~kg}$, et qui ne présentent pas de défaut agronomique jugé rédhibitoire. Une trentaine d'individus ont ainsi été vinifiés durant les trois années de sélection intermédiaire sur les deux sites. Pour l'ensemble des vins, l'évaluation sensorielle est réalisée grâce au plateau technique d'Agroscope, en mobilisant un jury entraîné d'une quinzaine de dégustateurs. En étape de sélection intermédiaire, cette épreuve est utilisée pour éliminer les individus dont les vins sont régulièrement jugés peu qualitatifs, ou pour départager des individus qui paraissent équivalents sur le plan agronomique. La Fig. 7 présente les résultats pour les 15 individus finalement sélectionnés pour l'étape finale, au moyen d'un descripteur global appelé 《impression générale », qui synthétise l'appréciation visuelle, olfactive et gustative des dégustateurs. Malgré les conditions de production sensiblement différentes entre Colmar et Pully, il y a une assez bonne relation entre les deux provenances, ce qui indique que l'effet génétique est prévalent, même pour ce critère intégrateur, très complexe. Un individu toutefois, bien jugé pour le vin de Pully et inversement pour le vin de Colmar, est particulièrement éloigné de la droite de régression. Il s'agit d'un individu très productif, pour lequel la régulation de charge pratiquée à Pully a plus particulièrement été bénéfique sur la qualité du vin.

\section{Conclusion}

Le programme de collaboration franco-suisse, porté par l'Inra et Agroscope, a permis de créer des populations de sélection avec des effectifs élevés, à partir de géniteurs complémentaires pour les résistances aux maladies, la précocité, la productivité et les profils des vins. Les premiers résultats de l'évaluation en sélection intermédiaire montrent que les descendants pyramidés sont dotés d'une résistance totale à l'oïdium et très élevée au mildiou. Une partie d'entre eux possède également une bonne résistance au black rot. Sur les plans agronomique et œnologique, ces individus sont caractérisés par une diversité qui laisse entrevoir un débouché pour une large gamme de vignobles. L'étape de 


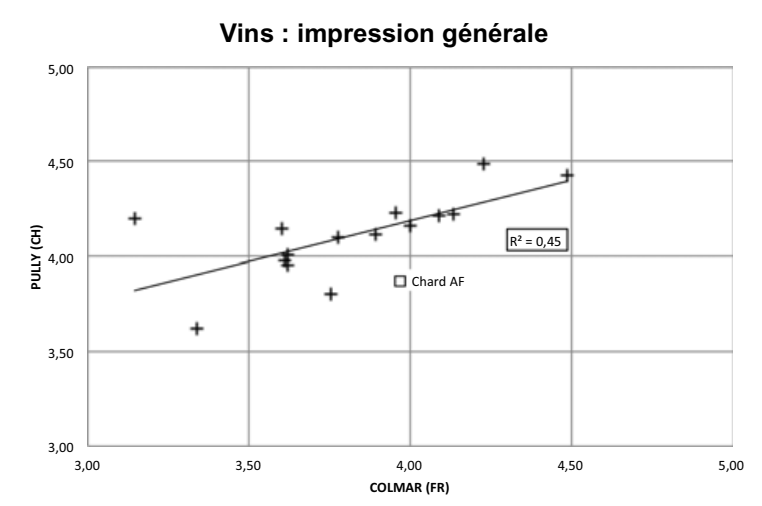

Figure 7. Relation entre la note de qualité globale des vins produits sur les dispositifs de Colmar et de Pully (Moyenne de trois années, 15 individus retenus pour la sélection finale SF, témoin Chardonnay $\mathrm{AF}$ )

sélection finale a démarré pour 15 co-obtentions, ouvrant la voie aux premières inscriptions au catalogue à l'horizon 2024-2025.

\section{Références}

[1] S. Tual, C. Lemarchand, M. Boulanger, N. LevêqueMorlais, S. Perrier, B. Clin, E. Rigaud, A.V. Guizard, M. Velten, I. Baldi, P. Lebailly, Innovations agronomiques 46, 136 (2015)

[2] R. Muthmann, The use of Plant Protection Products in the European Union (Eurostat, 2007)

[3] J.P. Butault, N. Delame, F. Jacquet, G. Ardet, NESE 35, 7 (2011)

[4] M. Blanck, C. Huyghe, Les certificats d'économie des produits phytopharmaceutiques (France Agricole, 2016)

[5] D. Boubals, Ann Amélior Plantes 6, 481 (1959)

[6] G. Staudt, H.H. Kassemeyer, Vitis 34, 225 (1995)

[7] L. Cadle-Davidson, Plant Dis. 92, 1577 (2008)

[8] P. Mestre, D. Merdinoglu, S. WiedemannMerdinoglu, A. Calonnec, L. Delière, F. Delmotte, Innovations Agronomiques 27, 37 (2013)
[9] E. Peressotti, S. Wiedemann-Merdinoglu, F. Delmotte, D. Bellin, G. Di Gaspero, R. Testolin, D. Merdinoglu, P. Mestre, BMC Plant Biol. 10, 147 (2010)

[10] A. Feechan, M. Kocsis, S. Riaz, W. Zhang, D.M. Gadoury, M.A. Walker, I.B. Dry, B. Reisch, L. CadleDavidson, Phytopathol. 105, 1104 (2015)

[11] D. Merdinoglu, C. Schneider, E. Prado, S. Wiedemann-Merdinoglu, P. Mestre, OENO One 52, 189 (2018)

[12] Organisation Internationale de la Vigne et du Vin, OIV, Resolution OIV-VITI 515 (2013)

[13] R. Eibach, E.M. Zyprian, L. Welter, R. Töpfer, Vitis 46, 120 (2007)

[14] C. Schneider, C. Onimus, E. Prado, V. Dumas, S. Wiedemann-Merdinoglu, M.A. Dorne, M.C. Lacombe, M.C. Piron, A. Umar-Faruk, E. Duchêne, P. Mestre, D. Merdinoglu, XII International Conference on Grapevine Breeding and Genetics (Bordeaux, France, 2018)

[15] A. Bouquet, 4ème conférence internationale de génétique de la vigne, Vérone, Italie, Vignevini 12, 141 (1986)

[16] K. Gindro, V. Alonso Villaverde, F. Voinesco, J.L. Spring, O. Viret, P.H. Dubuis, Plant Physiol. Biochem. 52, 140 (2012)

[17] J.L. Spring, K. Gindro, F. Voinesco, M. Jermini, M. Ferretti, O. Viret, Revue suisse Vitic., Arboric., Hortic. 45, 292 (2013)

[18] J.L. Spring, K. Gindro, F. Laprand, V. Zufferey, T. Verdenal, J. Rösti, L. Amiet, F. Lorenzini, P. Duruz, E Barmes, S Bailly, S. May, Y. Bonvin, R. Reymond, O. Viret, C. Carlen, Revue suisse Vitic., Arboric., Hortic. 50, 286 (2018)

[19] P. Blasi, S. Schnee, S. Wiedemann-Merdinoglu, E. Prado, S. Godard, P. Coste, C. Onimus, K. Gindro, C. Schneider, O. Viret, D. Merdinoglu, $6^{\text {th }}$ International Workshop of Grapevine Downy and Powdery Mildew (Bordeaux, France, 2010)

[20] F. Rex, I. Fechter, L. Hausmann, R. Töpfer, Theor. Appl. Genet. 127, 1667 (2014) 